\title{
Off the Mainstream: Advances in Neural Networks and Machine Learning for Pattern Recognition
}

\author{
Edmondo Trentin ${ }^{1}$. Friedhelm Schwenker ${ }^{2}$. \\ Neamat El Gayar ${ }^{3}$. Hazem M. Abbas ${ }^{4}$
}

Published online: 5 June 2018

(C) Springer Science+Business Media, LLC, part of Springer Nature 2018

This Special Issue (SI) originates from an event we organized in Ulm, Germany, in September 2016, namely the seventh IAPR TC3 Workshop on Artificial Neural Networks in Pattern Recognition (ANNPR2016) [18], sponsored by the International Association for Pattern Recognition (IAPR) and managed by its Technical Committee 3 on Neural Networks and Computational Intelligence ${ }^{1}$ (TC3). In the era of deep learning, ANNPR2016 aimed to create a common ground for researchers active specifically in applications to pattern recognition tasks of neural networks and other machine learning approaches. After the success of the workshop, and in the light of the exquisite scientific contribution of several presentations given therein, it came to our minds the idea of proposing a special issue of a relevant journal, to be based on extended versions of selected papers from ANNPR2016. The Editorial Board of Neural Processing Letters accepted the proposal, encouraging us to proceed with the initiative. At that point, we decided to extend the scope of the SI to an even broader audience by means of an open call for papers on the topic of "off-the-mainstream" approaches to

\footnotetext{
${ }^{1}$ See http://iapr-tc3.diism.unisi.it/.

$凶$ Edmondo Trentin

trentin@dii.unisi.it

Friedhelm Schwenker

friedhelm.schwenker@uni-ulm.de

Neamat El Gayar

elgayar.neamat@gmail.com

Hazem M. Abbas

hazem.abbas@eng.asu.edu.eg

1 DIISM, Università di Siena, Via Roma, 56, 53100 Siena, Italy

2 Institute of Neural Information Processing, Ulm University, James-Franck-Ring, 89081 Ulm, Germany

3 Faculty of Computers and Information, Cairo University, 5 Dr Ahmed Zewail Street, Orman, Giza 12613, Egypt

4 Department of Computer and Systems Engineering, Ain Shams University, 1 ElSarayat St., Abbassia, Cairo 11517, Egypt
} 
pattern recognition by means of neural nets and learning paradigms. The call received an enthusiastic response by the community. Eventually, this SI is the result of a selection of the best peer-reviewed submissions of both stems.

\section{The Need for "Off the Mainstream" Science}

"Mainstream science is about publishing what everyone else is publishing with very small changes. You'd better at least start off that way if you want to get tenure", the sociologist Rodney Stark said [21]. But "big ideas don't come to those who avoid risk", as John Bohannon added [3]. The area of neural networks and machine learning makes no exception to these ends. Mainstream topics, originally stemming from exciting breakthroughs (the "big ideas") that gradually become trends and end-up being mostly over-beaten publishing tracks, have characterized the scientific literature throughout the whole history of these research fields. A few, widely known instances of such (more or less recent) mainstream trends are:

- supervised support vector machine training (in both primal and dual)

- supervised multilayer perceptron training via regular backpropagation

- radial basis function networks

- Bayesian networks (either shallow or deep)

- deep feed-forward and convolutional neural networks

as well as the countless applications and studies on the approximation capabilities of the aforementioned machineries.

Based on these premises, this special issue invited paper submissions on real novel research developments in the areas of neural networks and learning machines that (i) were rooted in (or, aimed at) pattern recognition, and that, above all, (ii) did not follow in the footsteps of nowadays established trends. Preference over applications, theoretical analysis, and variants of established techniques, was therefore given to submissions that handed out fresh and innovative ideas, architectures, or algorithms, even if they presented research that were still in its infancy (e.g., possibly lacking of a complete investigation of their theoretical properties, or of a large-scale application). It is seen that, by definition, a detailed list of topics of interest to the present branch of "off the mainstream" science would contradict the very perspective of the present SI. Hence, let the papers published in the special issue speak for themselves.

\section{The SI: Organization and List of Papers}

In this section we provide the reader with an ordered list of the papers which the SI is built upon. A concise overview of the major themes addressed by each paper is given, as well. We organized the papers according to a taxonomy reflecting their nature. Such a taxonomy encompasses, in the order: (1) algorithms for structured and graphical data, (2) models of sequences and time series, (3) associative memories, (4) feature-oriented techniques, and (5) density-related algorithms. Some of the papers might as well fit more than one of these groupings: whenever it was the case, we assigned the article to the category whose state of advancement would benefit the most from the scientific contribution offered by the specific paper at hand.

1. Algorithms for structured and graphical data we open the special issue with a set of papers that covers a topic which is as timely as intriguing, namely learning and processing data that are structured as graphs (or, sequences of graphs). In their paper 
Multilayer Graph Node Kernels: Stacking while Maintaining Convexity, Luca Oneto, Nicolò Navarin, Alessandro Sperduti and Davide Anguita propose a multi-layer kernel for graphs, realizing the enhancement of established graph-kernels [9] by means of a deep learning strategy based on convex optimization. In the next paper, Time Series Prediction for Graphs in Kernel and Dissimilarity Spaces, Benjamin Paassen, Christina Goepfert and Barbara Hammer propose viable dissimilarity- or kernel-based regression techniques for the modeling and the prediction of the evolution of graphs (as a whole) over time. In the last paper of this first set, titled On the Impact of Using Utilities Rather than Costs for Graph Matching (an extension of their ANNPR2016 paper [16]), Kaspar Riesen, Andreas Fischer and Horst Bunke face the problem of exponential time complexity of the graph matching procedures that rely on the edit distance. A transformation process in proposed, aimed at converting the cost model (which underlies the efficient reformulation of the edit distance problem in terms of a linear sum assignment problem) into a utility model. In so doing, the integration of additional information in the assignment process is enabled, resulting in effective distance-based graph classifiers.

2. Models of sequences and time series the second group of papers revolves around models that are suitable to learning from sequences of input patterns and modeling time-series, possibly including applications to such areas as audio-visual signals, bioinformatics, and optical character recognition. The first paper in this grouping, namely A Temporal Dependency based Multi-modal Active Learning Approach for Audiovisual Event Detection (an extension of paper [23], presented at ANNPR2016) by Patrick Thiam, Sascha Meudt, Günther Palm and Friedhelm Schwenker, proposes a general transfer learning mechanism [7] which relies on a novel strategy for the automatic labeling of unlabeled samples in a partially supervised setup [19]. The proposed mechanism is then applied to several learners involved in a multi-modal event detection system fed with speech and video sequences. In the next paper, titled Dynamic Hybrid Random Fields for the Probabilistic Graphical Modeling of Sequential Data: Definitions, Algorithms, and an Application to Bioinformatics (extended version of the ANNPR2016 paper [4]), Marco Bongini, Antonino Freno, Vincenzo Laveglia and Edmondo Trentin formalize the notion of a dynamic extension to sequences of a probabilistic graphical model (the hybrid random field $[5,25]$ ) that subsumes Bayesian networks and Markov random fields [11], presenting parameter and structure learning algorithms and an application to the prediction of the disulfide bonding state of cysteines [28]. Then, Mohamed A. Radwan, Mahmoud Khalil and Hazem Abbas, in their paper Neural Networks Pipeline for Offline Machine Printed Arabic OCR (an extended version of their ANNPR2016 paper [15]) present a viable solution to the problem of Arabic optical characters recognition relying on a pipeline of three separate neural modules taking responsibility, respectively, for: (i) font size normalization, (ii) word segmentation into characters, and (iii) character recognition via a convolutional neural network. Results on Arabic characters drawn from the APTI data set [20] are presented and discussed. Finally, Witali Aswolinskiy, Felix Reinhart and Jochen Steil, in their paper Time Series Classification in Reservoir-and Model-Space (extended version of their ANNPR2016 paper [2]), present a large-scale experimental investigation of the prediction of both univariate and multivariate time series relying on reservoir computing [13]. Two approaches are thoroughly compared, namely reservoir-space and model-space variants of the basic reservoir machine. A combination of the two emerges as a viable and fruitful advancement over the raw variants, as well, at least in the univariate case.

3. Associative memories papers in this third grouping revolve around the notion of associative memory. In Theoretical Foundations for the Alpha-Beta Associative Memories - 10 
years of Derived Extensions, Models, and Applications, Cornelio Yáñez-Márquez, Itzamá López-Yáñez, Mario Aldape-Pérez, Oscar Camacho-Nieto, Amadeo-José Argélles-Cruz and Yenny Villuendas-Rey provide a thorough mathematical formalization of the AlphaBeta associative memory, a variant of the bidirectional associative memory (BAM) [12], offering a proper contextualization of the topic in the form of a survey of a long line of publications that empirically relied on the Alpha-Beta model throughout the last decade. The other paper in this batch, On the Weighted pseudo almost periodic solution for BAM networks with delays, written by Boudour Ammar, Hajer Brahmi, and Farouk Cherif, introduces a class of neural networks belonging to the broader class of BAMs. These machines are characterized by time-varying weights and continuously distributed delays. Sufficient conditions for the existence (and, uniqueness) of weighted pseudo almost-periodic solutions for this family of BAMs are presented, along with corroborating numerical simulations.

4. Feature-oriented techniques the papers in this set face a fundamental, yet often underrated issue, that is the relevance of adequate feature extraction, representation, and selection. In The influence of multi-class feature selection on the prediction of diagnostic phenotypes (extended version of [17], presented at ANNPR2016), Ludwig Lausser, Robin Szekely, Lyn-Rouven Schirra, and Hans A. Kestler propose and investigate empirically two new networks for feature selection within a multi-class classifier trained from a limited amount of high-dimensional data. The resulting machine is positively evaluated on a number of experiments in predicting diagnostic phenotypes from gene expression profiles [22]. In Ultra-sparse Classifiers through Minimizing the VC dimension in the Empirical Feature Space, Jayadeva, Mayank Sharma, Sumit Soman, and Himanshu Pant propose an empirical feature space (EFS) [1] version of their minimal complexity machine (MCM), that is capable of both minimizing the model complexity and improving its sparsity. This involves an ad-hoc least squares version of the MCM in the EFS. Experimental results underline the effectiveness of the approach.

5. Density-related algorithms the last two papers of the special issue cope with techniques that focus on the density of the data in the feature space. In the extended version to his ANNPR2016 paper [24], titled Soft-constrained Neural Networks for Nonparametric Density Estimation, Edmondo Trentin introduces a robust non-parametric connectionist technique for the estimation of probability density functions. The approach relates loosely to $[26,27]$ but accounts for the issue of having a feed-forward neural network to respect Kolmogorov's axioms of probability. The experiments (involving univariate and multivariate data drawn from complex distributions) show a statistically significant improvement over the established techniques. The next (and, last) paper of the special issue is Density Based Cluster Growing via Dominant Sets written by Jian Hou, Xu E, Weixue Liu as an extension to their ANNPR 2016 paper [10]. This article discusses a twostep clustering algorithm aimed at overcoming the drawbacks of traditional approaches by combining the merits of dominant sets clustering [6] and density based clustering [8]. In particular, a density threshold based cluster growing step is applied, and followed by a second growing step which exploits the density relationship between neighboring data.

Acknowledgements The present Special Issue is dedicated to the memory of our fellow scientist Ilaria Castelli, PhD, who served as a reviewer for this issue and tragically died at age 34. The ANNPR2016 Workshop (that originated the idea of this special issue in the first place) was co-sponsored by IAPR and its TC 3 , that are gratefully acknowledged. The partnership between the Universities of Ulm and Siena was partially granted under the Vigoni Program, managed by the Ateneo Italo-Tedesco/Deutsch-Italienisches Hochschulzentrum and co-funded by German DAAD and Italian MIUR. We would express our gratitude to the Editors-in-Chief of Neural Processing Letters, who supported our project. We are also thankful to the Springer editorial staff and 
to the many reviewers who contributed, timely and conscientiously, to the fulfillment of the present SI. Finally, we are grateful to all the authors of the papers embraced herein, who reacted promptly and enthusiastically to our editorial initiative with contributions of such a high quality.

\section{References}

1. Abe S (2007) Sparse least squares support vector training in the reduced empirical feature space. Pattern Anal Appl 10:203-214

2. Aswolinskiy W, Reinhart RF, Steil JJ (2016) Time series classification in reservoir- and model-space: a comparison. In: Schwenker et al. [18], pp 197-208

3. Bohannon J (2008) Courting controversy: Out of the mainstream. Science 360(6386):42-50

4. Bongini M, Laveglia V, Trentin E (2016) A hybrid recurrent neural network/dynamic probabilistic graphical model predictor of the disulfide bonding state of cysteines from the primary structure of proteins. In: Schwenker et al. [18], pp 257-268

5. Bongini M, Trentin E (2016) Towards a novel probabilistic graphical model of sequential data: a solution to the problem of structure learning and an empirical evaluation. In: Mana et al. [14], pp 82-92

6. Bulò SR, Pelillo M (2017) Dominant-set clustering: a review. Eur J Oper Res 262(1):1-13

7. Dietterich TG, Pratt L, Thrun S (1997) Special issue on inductive transfer. Mach Learn 28(1):215-220

8. Ester M, Kriegel HP, Sander J, Xu X (1996) A density-based algorithm for discovering clusters in large spatial databases with noise. In: Proceedings of the second international conference on knowledge discovery and data mining, KDD’96, pp 226-231. AAAI Press

9. Gaertner T (2003) A survey of kernels for structured data. SIGKDD Explor Newsl 5(1):49-58

10. Hou J, Liu W, Xu E (2016) Density based clustering via dominant sets. In: Schwenker et al. [18], pp 80-91

11. Koller D, Friedman N (2009) Probabilistic graphical models: principles and techniques. The MIT Press, Cambridge

12. Kosko B (1988) Bidirectional associative memories. IEEE Trans Syst Man Cybernet 18(1):49-60

13. Lukosevicius M, Jaeger H, Schrauwen B (2012) Reservoir computing trends. KI Künstliche Intelligenz 26(4):365-371

14. Mana N, Schwenker F, Trentin E (eds) (2012) Artificial neural networks in pattern recognition. Proceedings of the 5th INNS IAPR TC 3 GIRPR workshop, ANNPR 2012, Trento, Italy, September 17-19, 2012. Lecture Notes in Computer Science, vol 7477. Springer

15. Radwan MA, Khalil MI, Abbas HM (2016) Predictive segmentation using multichannel neural networks in Arabic OCR system. In: Schwenker et al. [18], pp 233-245

16. Riesen K, Fischer A, Bunke H (2016) Approximation of graph edit distance by means of a utility matrix. In: Schwenker et al. [18], pp 185-194

17. Schirra L, Schmid F, Kestler HA, Lausser L (2016) Interpretable classifiers in precision medicine: feature selection and multi-class categorization. In: Schwenker et al. [18], pp 105-116

18. Schwenker F, Abbas HM, Gayar NE, Trentin E (eds) (2016) Artificial Neural Networks in Pattern Recognition. Proceedings of the 7th IAPR TC3 workshop, ANNPR 2016, Ulm, Germany, September 28-30, 2016. Lecture Notes in Computer Science, vol 9896. Springer

19. Schwenker F, Trentin E (2014) Pattern classification and clustering: a review of partially supervised learning approaches. Pattern Recogn Lett 37:4-14

20. Slimane F, Ingold R, Kanoun S, Alimi A, Hennebert J (2009) Database and evaluation protocols for Arabic printed text recognition. Technical Report 296-09-01, University of Fribourg, Department of Informatics. http://www.hennebert.org/download/publications/unifr-tech-report-296-0901_database_and_evaluation_protocols_for_arabic_printed_text_recognition_apti.pdf. Accessed 05 Mar 2018

21. Stark R, Iannaccone L, Finke R (1996) Religion, science, and rationality. Am Econ Rev 86(2):433-37

22. Tarca AL et al (2013) Strengths and limitations of microarray-based phenotype prediction: lessons learned from the improver diagnostic signature challenge. Bioinformatics 29(22):2892-2899

23. Thiam P, Meudt S, Schwenker F, Palm G (2016) Active learning for speech event detection in HCI. In: Schwenker et al. [18], pp 285-297

24. Trentin E (2016) Soft-constrained nonparametric density estimation with artificial neural networks. In: Schwenker et al. [18], pp 68-79

25. Trentin E, Bongini M (2016) Towards a novel probabilistic graphical model of sequential data: fundamental notions and a solution to the problem of parameter learning. In: Mana et al. [14], pp 72-81 
26. Trentin E, Freno A (2009) Unsupervised nonparametric density estimation: a neural network approach. In: International joint conference on neural networks, IJCNN 2009, Atlanta, Georgia, USA, 14-19 June 2009, pp 3140-3147

27. Trentin E, Lusnig L, Cavalli F (2018) Parzen neural networks: fundamentals, properties, and an application to forensic anthropology. Neural Netw 97:137-151

28. Wuskal SM, Holbrook SR, Kim SH (1990) Prediction of the disulfide-bonding state of cysteine in proteins. Protein Eng Des Sel 3(8):667-672

Publisher's Note Springer Nature remains neutral with regard to jurisdictional claims in published maps and institutional affiliations. 